\title{
Reply to the commentary by Sarode and Sarode on "Genetic toxicology and toxicokinetics of arecoline and related areca nut compounds: an updated review"
}

\author{
Nuno G. Oliveira' (1) - Daniela L. Ramos ${ }^{1,2} \cdot$ Ricardo Jorge Dinis-Oliveira $^{3,4,5}$ (]) \\ Received: 19 March 2021 / Accepted: 1 April 2021 / Published online: 12 April 2021 \\ (c) The Author(s), under exclusive licence to Springer-Verlag GmbH Germany, part of Springer Nature 2021
}

We thank Drs S.C. Sarode and G.S. Sarode for their comments and interest on our recent review entitled "Genetic toxicology and toxicokinetics of arecoline and related areca nut compounds: an updated review" (Oliveira et al. 2021). Arecoline is the primary active ingredient found in areca nut (AN) which is chewed by approximately $10 \%$ of human population. As addressed in our review, a variety of chemicals are present in the several types of AN-containing products. The AN alkaloids and other compounds along with the different arecoline metabolites and nitrosation products identified in the saliva of consumers render this type of exposure a very complex toxicological issue. The commentary Sarode and Sarode (2021) highlighted that only a few clinical studies described in the monograph from 2004 of the International Agency for Research on Cancer (IARC 2004) mentioned the exposure to AN alone. Indeed, additional clinical studies focusing on individuals exposed to $\mathrm{AN}$ alone are still needed. Nevertheless, it is also important to note that AN,

Nuno G. Oliveira

ngoliveira@ff.ulisboa.pt

Ricardo Jorge Dinis-Oliveira

ricardinis@med.up.pt

1 Research Institute for Medicines (iMed.ULisboa), Faculty of Pharmacy, Universidade de Lisboa, Lisbon, Portugal

2 Pharmacy Service, Centro Hospitalar Universitário Lisboa Norte, EPE, Hospital de Santa Maria, Lisbon, Portugal

3 Department of Public Health and Forensic Sciences, and Medical Education, Faculty of Medicine, University of Porto, Porto, Portugal

4 UCIBIO, REQUIMTE, Laboratory of Toxicology, Department of Biological Sciences, Faculty of Pharmacy, University of Porto, Porto, Portugal

5 Department of Sciences, IINFACTS, Institute of Research and Advanced Training in Health Sciences and Technologies, University Institute of Health Sciences (IUCS), CESPU, CRL, Gandra, Portugal as well as Betel Quid (BQ) with and without added tobacco were all classified by IARC in Group 1 -carcinogenic to humans (IARC 2004, 2012).

Overall, the major points raised in the commentary are somehow related to the clinical and translational significance of this topic. While we consider that these aspects are of utmost importance to establish a causal link between exposure to AN and cancer, it should be emphasized that the scope of our work was primarily to review in a comprehensive manner the available genetic toxicology data of arecoline and related compounds present in AN. These chemicals, particularly arecoline and its active metabolites are indeed determinant in the context of AN exposure, as we described in our article. Recently, an additional path was given for the translational medicine. Indeed, accordingly to the abstract, arecoline induced epithelial-mesenchymal transformation and increased the metastatic capability of oral squamous cell carcinoma (OSCC) by upregulating inflammatory cytokines such as serum amyloid A1 (Ren et al. 2021). Also, already this year, a very interesting study analyzed OSCC human samples for arecoline and its metabolites and further highlighted the importance of arecoline $\mathrm{N}$-oxide in the initial stages of carcinogenesis, by inducing inflammatory and oxidative dysregulation (Nithiyanantham et al. 2021). In our work, we also intended to review many of the toxicokinetic aspects of these compounds and to discuss the different mechanisms possibly involved in arecoline's genetic toxicity, presenting also the novel strategies to increase the understanding in this field. All the knowledge has been generated in several in vitro and in vivo studies by many authors through the years, and was revisited and systematized in the review. In our opinion, the understanding of the mode of action of a given genotoxic agent is critical to provide evidence supporting its role as a putative carcinogen being this also relevant in a translational context and risk assessment.

The recent IARC evaluation of arecoline in volume 128 of the IARC monographs (IARC 2020) was also mentioned 
in the commentary by Sarode and Sarode (2021). Last year in October-November a working group of scientists invited by IARC finalized the evaluations on the carcinogenicity of acrolein, crotonaldehyde and arecoline. The results from this evaluation process were recently published online in The Lancet Oncology (IARC 2021). In this summary article, available approximately one month after our article was published online in its final version, arecoline was classified in Group 2B-“Possibly carcinogenic to humans". In our review we have already mentioned that arecoline was considered in the group of agents recommended for evaluation with high priority by the IARC Monographs Advisory Group (IARC 2019), and publications mentioned above and in our review are giving strength to recent IARC decisions. The summary article identified several genotoxic features displayed by arecoline in different endpoints, all of them addressed in our review. These studies support the "strong" evidence that arecoline exhibits key characteristics of a carcinogen (IARC 2021). The summary article also mentioned the "limited" evidence for carcinogenicity from in vivo studies with experimental animals that along with the absence of data from human studies with arecoline alone lead to the overall classification of arecoline in Group 2B. In conclusion, we acknowledge the authors of this comment for the opportunity to further reinforce the importance of arecoline and related compounds and the need of further studies, namely clinical ones.

\section{Declarations}

Conflict of interest The authors declare that they have no conflict of interest.

\section{References}

IARC (2004) Betel-quid and areca-nut chewing and some areca-nut derived nitrosamines. IARC Monogr Eval Carcinog Risks Hum 85:1-334

IARC (2012) Personal habits and indoor combustions. Volume $100 \mathrm{E}$. A review of human carcinogens. IARC Monogr Eval Carcinog Risks Hum 100(Pt E):1-538.

IARC (2019) Report of the advisory group to recommend priorities for the IARC monographs during 2020-2024

IARC (2020) Volume 128: acrolein, crotonaldehyde, and arecoline. IARC Working Group. Lyon, France; Oct 29-Nov 13, 2020; IARC Monogr Identif Carcinog Hazards Hum (in press)

IARC (2021) Carcinogenicity of acrolein, crotonaldehyde, and arecoline. Lancet Oncol 22:19-20

Nithiyanantham S, Arumugam S, Hsu HT, Chung CM, Lee CP, Tsai MH, Yeh KT, Luo SY, Ko YC (2021) Arecoline N-oxide initiates oral carcinogenesis and arecoline $\mathrm{N}$-oxide mercapturic acid attenuates the cancer risk. Life Sci 271:119156

Oliveira NG, Ramos DL, Dinis-Oliveira RJ (2021) Genetic toxicology and toxicokinetics of arecoline and related areca nut compounds: an updated review. Arch Toxicol 95:375-393

Ren H, He G, Lu Z, He Q, Li S, Huang Z, Chen Z, Cao C, Wang A (2021) Arecoline induces epithelial mesenchymal transformation and promotes metastasis of oral cancer via SAA1 expression. Cancer Sci. https://doi.org/10.1111/cas.14866 (in press)

Sarode SC, Sarode GS (2021) Comment on "Genetic toxicology and toxicokinetics of arecoline and related areca nut compounds: an updated review. Arch Toxicol. https://doi.org/10.1007/ s00204-021-03027-x

Publisher's Note Springer Nature remains neutral with regard to jurisdictional claims in published maps and institutional affiliations. 